Relations industrielles

Industrial Relations

\title{
The Impact of Computers on Management, par Charles A. Myers (Editor), The MIT Press, Massachusetts, 1963, 310 pp.
}

\section{Pierre Brien}

Volume 24, numéro 3, 1969

URI : https://id.erudit.org/iderudit/028060ar

DOI : https://doi.org/10.7202/028060ar

Aller au sommaire du numéro

Éditeur(s)

Département des relations industrielles de l'Université Laval

ISSN

0034-379X (imprimé)

1703-8138 (numérique)

Découvrir la revue

Citer ce compte rendu

Brien, P. (1969). Compte rendu de [The Impact of Computers on Management, par Charles A. Myers (Editor), The MIT Press, Massachusetts, 1963, 310 pp.] Relations industrielles / Industrial Relations, 24(3), 644-645.

https://doi.org/10.7202/028060ar

Tous droits réservés (C) Département des relations industrielles de l'Université Laval, 1969
Ce document est protégé par la loi sur le droit d'auteur. L'utilisation des services d'Érudit (y compris la reproduction) est assujettie à sa politique d'utilisation que vous pouvez consulter en ligne.

https://apropos.erudit.org/fr/usagers/politique-dutilisation/ 
Gourand, Henry, Jabot, Morel, Muller, Ogus, Pinelli, Schaller, ainsi que 1'Association française des ingénieurs et chefs d'entretien.

Il nous faut dès à présent abandonner l'acceptation restrictive de l'entretien considéré comme un service de réparation. On doit le concevoir non plus comme un palliatif mais plutôt insister sur l'aspect prévention de ce service. Il faut reconsidérer la place de ce service dans l'organigramme de l'entreprise.

A l'intérieur même du service, la répartition classique des tâches par technique est dépassée, il faut chercher à regrouper ces tâches par fonction. Les études sur les problèmes à résoudre, la préparation du travail, l'exécution du travail, le contrôle et la gestion de la main-d'oeuvre seront les fonctions du service d'entretien. Et le «bureau technique » sera le cerveau et le système nerveux de ce service. De plus, on suggère une autorité fonctionnelle sur toutes les autres divisions à ce bureau, de même qu'on énumère les différents « outils de travail» dont il devra disposer pour être efficace.

Une fois le côté technique assuré, il faut le concilier avec le côté administratif. Cet ensemble d'opérations technico-administratives est appelé «la préparation du travail ». De nombreuses expériences prouvent que cette planification est nécessaire, surtout si l'on a affaire à la mise hors service d'une partie importante de l'installation.

L'étude énonce ensuite certains facteurs qui concourrent à l'efficacité du service d'entretien ainsi que la façon d'en mesurer la rentabilité et finalement donne quelques recettes pratiques et simples pour en diminuer le coût.

Signalons également que le présent volume donne un excellent aperçu des possibilités et de la mise en pratique de la méthode P.E.R.T. (Program Evaluation and Review Technique) sur lequel il existe déjà une abondante littérature.

Dans la deuxième partie du volume, on s'interroge à savoir s'il vaut mieux avoir son propre personnel au service d'entretien et dans quel cas sera-t-il préférable de faire appel à la main-d'oeuvre extérieure? Quels sont les moyens les plus efficaces pour arriver à un «service absorbé » d'entretien? Du côté des stocks ou du matériel, quoi et quand acheter ? Quelle est la méthode pour trouver le niveau optimum des stocks? V'oilà autant de questions auxquelles les auteurs s'appliquent à répondre.

Enfin en terminant, on insiste sur la nécessité d'un entretien préventif comme étant la meilleure garantie d'avoir un «service absorbé ». Encore là, les suggestions et les étapes à suivre sont clairement expliquées.

Bref, ce volume apporte quantité d'idées nouvelles sur un « service» de l'entreprise, i.e. l'entretien qui, jusqu'à ces dernières années, était demeuré en reste mais qui, une fois réorganisé et repensé, peut constituer un premier mouvement vers une plus grande rentabilité.

\section{Michel BERNARD.}

The Impact of Computers on Management, par Charles A. Myers (Editor), The MIT Press, Massachusetts, 1963, $310 \mathrm{pp}$.

Cette publication est le fruit d'une conférence multidisciplinaire. C. A. Myers regroupe les sept exposés et fait état des discussions qui s'en suivirent.

1. - «The Impact of Information Technology on Organizational Control », par Thomas L. Whisler (Un. of Chicago). Cet auteur se demande quels seront les effets de l'informatique sur les structures de contrôle au sein des organisations. Quel en sera l'impact sur le travail de certains individus? Est-ce qu'elle accentuera le caractère routinier des tâches ? Conduira-t-elle à des modifications du degré d'autorité dans les différents postes? Enfin, est-ce que les machines en viendront à partager le contrôle des activités avec l'homme?

2. - «Computers and Organization Structure in Life-Insurance Firms: The External and Internal Economic Environment », par George E. Delehanty, (Northwestern Un.). L'auteur rapporte qu'il n'existe pas de pressions compétitives qui incitent sérieusement les compagnies d'assurances à se servir des « ordinateurs pour réduire leurs coûts d'opérations. En d'autres termes, ils ont eu très peu d'effets sur l'industrie. Cette situation peut s'expliquer par les nombreux changements dans les structures 
qu'impose l'introduction des systèmes électroniques. Au cours des prochaines années les besoins de main-d'oeuvre diminueront et seront compensés par les ordinateurs qui seront devenus porportionnellement moins dispendieux.

3. - « Tasks, Organization Structures, and Computer Programs », par David Klahr et Harold J. Leavitt (Carnegie Institute of Technology). Les auteurs ananlysent les relations entre trois variables importantes: les fonctions, les structures de l'organisation et les programmes sortis des ordinateurs. Ils croient qu'une nouvelle vague d'interactions apparaîtra entre les organisations et l'introduction de systèmes électroniques de plus en plus perfectionnés, puisque ces derniers affectent maintenant le partage des responsabilités au niveau des cadres supérieurs.

4. - « Implications of On-Line, RealTime systems for Managerial DecisionMaking », par Donald C. Carroll (MIT). Selon l'auteur l'informatique influence sérieusement la prise de décision qui, elle-même, affecte toute l'organisation. Son argument repose sur trois avantages qu'offre la technologie: la facilité d'accumuler des données de base, la capacité d'analyser ces données au moment de prendre une décision et la possibilité de solutionner les problèmes du couple homme-machine.

5. - « Computers and Profit Centers $\gg$ par John Dearden (Harvard Un.). L'auteur analyse l'effet de l'informatique sur les entreprises dont la profitabilité repose sur les grandes divisions. Les ordinateurs vont permettre d'obtenir une information plus précise plus rapidement et vont contribuer à déterminer les objectifs de profitabilité. Il semble cependant qu'ils ne faciliteront pas le contrôle des opérations divisionnelles par la haute direction.

6. - - The Total-Systems Concept : Its Implications for Management $\gg$ par John A. Beckett (Un. of New Hampshire). L'auteur traite de l'administration par l'automation. Il en souligne les facteurs d'accélération et insiste sur les préparations complexes à ce mode d'administration. De plus, il analyse la place de l'homme et des machines aux divers niveaux de la pyramide de l'autorité hiérarchique et de conseil.
7. - « Changes in Management Environment and their Effects Upon Value » par Charles R. DeCarlo (IBM). Cette dernière conférence traite des changements possibles d'attitudes chez les dirigeants par suite de l'introduction des ordinateurs. L'auteur souligne la professionnalisation plus poussée de la direction des entreprises, et insiste sur la nécessité d'une formation permanente des cadres.

\section{Pierre BRIEN}

Lexique de l'information, par J. Guilhaumou, Entreprise moderne d'édition, Paris, 1969, 121 pp.

A une période où l'informatique fait son entrée dans des domaines de plus en plus variés et nombreux, les personnes intéressées aux relations du travail, spécialement les directeurs de personnel et les directeurs des relations industrielles, ne peuvent demeurer indifférentes à cette technique. Pour eux et pour tous ceux concernés, le Lexique de l'informatique peut être un instrument de travail pratique, permettant d'éviter la confusion des termes trop souvent présentés dans bien des discussions et rapports.

Ce n'est pas avec la prétention d'avoir constitué un dictionnaire de tous les mots pouvant être utilisés dans le traitement de l'information que l'auteur présente son ouvrage. Il s'agit plutôt d'un « guide pratique pour l'usage des termes spécifiques de ce domaine d'activité, et des mots courrants qui y sont utilisés, dans un sens particulier $»$. Ce lexique de l'informatique définit au moins cinq cent cinquante termes ce qui lui permet de répondre aux exigences des profanes et aussi de bien des techniciens de cette spécialité. Les principales sources d'information consultées furent les travaux du Comité de vocabulaire de la firme IBM-France, pour laquelle l'auteur travaille, des projets de l'ISO (Organisation internationale de normalisation) et des documents du CCITT (Comité consultatif international télégraphique et téléphonique). D'autres documents de langue anglaise furent également utilisés tels que l'american Standard Vocabulary for information Processing publié par l'USAST (United States American Standard Institute) et le Vocabulary of Information Processing de l'IFTP-ICC. Ceci explique d'ailleurs que certains termes 